TRANSACTIONS OF THE

AMERICAN MATHEMATICAL SOCIETY

Volume 359, Number 10, October 2007, Pages 5019-5034

S 0002-9947(07)04215-8

Article electronically published on April 24, 2007

\title{
SIGMA FUNCTION SOLUTION OF THE INITIAL VALUE PROBLEM FOR SOMOS 5 SEQUENCES
}

\author{
A. N. W. HONE
}

\begin{abstract}
The Somos 5 sequences are a family of sequences defined by a fifth order bilinear recurrence relation with constant coefficients. For particular choices of coefficients and initial data, integer sequences arise. By making the connection with a second order nonlinear mapping with a first integral, we prove that the two subsequences of odd/even index terms each satisfy a Somos 4 (fourth order) recurrence. This leads directly to the explicit solution of the initial value problem for the Somos 5 sequences in terms of the Weierstrass sigma function for an associated elliptic curve.
\end{abstract}

\section{INTRODUCTION}

The subject of this article is the bilinear recurrence relation

$$
\tau_{n+3} \tau_{n-2}=\tilde{\alpha} \tau_{n+2} \tau_{n-1}+\tilde{\beta} \tau_{n+1} \tau_{n},
$$

where $\tilde{\alpha}$ and $\tilde{\beta}$ are constant parameters, which is known as the Somos 5 recurrence $\left[12,24,28\right.$. Somos 5 sequences are defined by specifying initial data $\tau_{0}, \tau_{1}, \tau_{2}$, $\tau_{3}, \tau_{4}$ together with the values of the coefficients $\tilde{\alpha}, \tilde{\beta}$, and when all of these are suitably chosen, then integer sequences can result. For example, when

$$
\tau_{0}=\tau_{1}=\tau_{2}=\tau_{3}=\tau_{4}=1, \quad \tilde{\alpha}=\tilde{\beta}=1,
$$

then the integer sequence

$$
1,1,1,1,1,2,3,5,11,37,83,274,1217,6161,22833, \ldots
$$

results. Traditionally the latter is referred to as the Somos 5 sequence, but we will follow the terminology of 24, 29] and refer to this specific sequence as Somos (5).

In recent work 13, we have considered fourth order quadratic recurrences of the form

$$
\tau_{n+2} \tau_{n-2}=\alpha \tau_{n+1} \tau_{n-1}+\beta\left(\tau_{n}\right)^{2},
$$

where $\alpha$ and $\beta$ are constant parameters. Sequences that satisfy such fourth order recurrences are known as Somos 4 sequences [24, 29, and they originally arose in the theory of elliptic divisibility sequences [33, 34, 25]. In that context, both the parameters $\alpha, \beta$ and the iterates $\tau_{n}$ are usually integers, in which case the sequences have applications in number theory, as they provide a potential source of large prime numbers [8, 9. Moreover Somos 4 sequences provide a simple example

Received by the editors February 9, 2005 and, in revised form, September 15, 2005.

2000 Mathematics Subject Classification. Primary 11B37, 33E05; Secondary 37J35.

Key words and phrases. Integer sequences, elliptic curves. 
of the Laurent phenomenon: taking the initial data $\tau_{0}, \tau_{1}, \tau_{2}, \tau_{3}$ and the parameters $\alpha, \beta$ as variables, all subsequent terms $\tau_{n}$ for $n \geq 4$ in the sequence are Laurent polynomials (rather than just rational functions) in these variables. Fomin and Zelevinsky have proved that this remarkable "Laurentness" property is shared by a variety of other recurrences in one and more dimensions, including the Somos 5 recurrences (1.1), and this has applications in combinatorics and commutative algebra (see 11 and references). Although Somos sequences are often defined over $\mathbb{Q}$, here we will work over $\mathbb{C}$ and occasionally indicate when our results restrict to yield rationals or integers.

Morgan Ward first introduced elliptic divisibility sequences [33, 34, a family of antisymmetric sequences with $a_{n}=-a_{-n}$ defined by recurrences of the form

$$
a_{n+2} a_{n-2}=\left(a_{2}\right)^{2} a_{n+1} a_{n-1}-a_{1} a_{3}\left(a_{n}\right)^{2},
$$

by considering sequences of rational points $n P$ on an elliptic curve $E$ over $\mathbb{Q}$ (see also [10, 25]). To obtain integer sequences of this kind it is required that

$$
a_{0}=0, \quad a_{1}=1, \quad a_{2}, a_{3}, a_{4} \in \mathbb{Z} \quad \text { with } \quad a_{2} \mid a_{4},
$$

and then it turns out that the subsequent terms of the sequence satisfy the remarkable divisibility property

$$
a_{n} \mid a_{m} \quad \text { whenever } \quad n \mid m .
$$

These integrality and divisibility properties can be said to rest on the fact that the terms of an elliptic divisibility sequence satisfy the Hankel determinant relation

$$
a_{n+m} a_{n-m}=\left|\begin{array}{cc}
a_{m} a_{n-1} & a_{m-1} a_{n} \\
a_{m+1} a_{n} & a_{m} a_{n+1}
\end{array}\right|,
$$

for all $m, n \in \mathbb{Z}$. Starting from the Hankel determinant formula, with initial data of the form (1.6), it is easy to prove by induction that all $a_{n}$ for $n \geq 0$ are integers with the divisibility property (1.7). Although with $a_{0}=0$ there is potentially the problem of dividing by zero, it turns out that these sequences are consistently extended to all $n \in \mathbb{Z}$.

As we explain below, the formula (1.8) itself is most easily proved by making use of Ward's explicit expression

$$
a_{n}=\frac{\sigma(n \kappa)}{\sigma(\kappa)^{n^{2}}}
$$

for the general term of the sequence in terms of the Weierstrass sigma function associated with the curve $E$, and then applying the addition formula

$$
\frac{\sigma(z+\kappa) \sigma(z-\kappa)}{\sigma(z)^{2} \sigma(\kappa)^{2}}=\wp(\kappa)-\wp(z)
$$

or its immediate consequence,

$$
\begin{aligned}
& \sigma(c+d) \sigma(c-d) \sigma(a+b) \sigma(a-b) \\
&-\sigma(b+d) \sigma(b-d) \sigma(a+c) \sigma(a-c) \\
&+\sigma(b+c) \sigma(b-c) \sigma(a+d) \sigma(a-d)=0,
\end{aligned}
$$

which is called the three-term equation (see $\S 20.53$ in [35]). Ward's elliptic divisibility sequences are just special cases of Somos 4 sequences. The following theorem was proved in 13 . 
Theorem 1.1. The general solution of the Somos 4 recurrence relation (1.4) takes the form

$$
\tau_{n}=A B^{n} \frac{\sigma\left(z_{0}+n \kappa\right)}{\sigma(\kappa)^{n^{2}}},
$$

where $\kappa$ and $z_{0}$ are non-zero complex numbers, the constants $A$ and $B$ are given by

$$
A=\frac{\tau_{0}}{\sigma\left(z_{0}\right)}, \quad B=\frac{\sigma(\kappa) \sigma\left(z_{0}\right) \tau_{1}}{\sigma\left(z_{0}+\kappa\right) \tau_{0}},
$$

and $\sigma(z)=\sigma\left(z ; g_{2}, g_{3}\right)$ denotes the Weierstrass sigma function of an associated elliptic curve

$$
E: \quad y^{2}=4 x^{3}-g_{2} x-g_{3} .
$$

The values $\kappa, z_{0}$ and the invariants $g_{2}, g_{3}$ are precisely determined from the initial data $\tau_{0}, \tau_{1}, \tau_{2}, \tau_{3}$ and the parameters $\alpha, \beta$, where the latter are given as elliptic functions of $\kappa$ by

$$
\alpha=\wp^{\prime}(\kappa)^{2}, \quad \beta=\wp^{\prime}(\kappa)^{2}(\wp(2 \kappa)-\wp(\kappa)) .
$$

The terms of a Somos 4 sequence correspond to the sequence of points $P_{0}+n P$ on the curve $E$, with $z_{0}+n \kappa$ being the associated sequence of points on the Jacobian. The elliptic divisibility sequences arise when $P_{0} \rightarrow \infty$ (or equivalently $z_{0} \rightarrow 0$ with $\tau_{0} \rightarrow 0$ ). To illustrate the effectiveness of such explicit formulae for these sequences in terms of Weierstrass functions, we can mention two corollaries of the above theorem.

Corollary 1.2. The terms of a Somos 4 sequence satisfy the Hankel determinant formula

$$
\tau_{n+m} \tau_{n-m}=\left|\begin{array}{ll}
a_{m} \tau_{n+1} & a_{m-1} \tau_{n} \\
a_{m+1} \tau_{n} & a_{m} \tau_{n-1}
\end{array}\right|
$$

for all $m, n \in \mathbb{Z}$, where $a_{m}=\sigma(m \kappa) / \sigma(\kappa)^{m^{2}}$ are the terms of an associated elliptic divisibility sequence.

Proof. Substituting the explicit expressions (1.9) and (1.12) for $a_{n}$ and $\tau_{n}$ into (1.16), setting $z=z_{0}+n \kappa$ and using (1.10) yields

$$
\begin{aligned}
& \frac{\sigma(\kappa)^{2 m^{2}+2 n^{2}}}{A^{2} B^{2 n} \sigma(m \kappa)^{2} \sigma(z)^{2}}\left\{\tau_{n+m} \tau_{n-m}-\left(a_{m}\right)^{2} \tau_{n+1} \tau_{n-1}+a_{m+1} a_{m-1}\left(\tau_{n}\right)^{2}\right\} \\
&= \frac{\sigma(z+m \kappa) \sigma(z-m \kappa)}{\sigma(z)^{2} \sigma(m \kappa)^{2}}-\frac{\sigma(z+\kappa) \sigma(z-\kappa)}{\sigma(z)^{2} \sigma(\kappa)^{2}}+\frac{\sigma((m+1) \kappa) \sigma((m-1) \kappa)}{\sigma(\kappa)^{2} \sigma(m \kappa)^{2}} \\
&=\wp(m \kappa)-\wp(z)-\wp(\kappa)+\wp(z)+\wp(\kappa)-\wp(m \kappa)=0 .
\end{aligned}
$$

Corollary 1.3. The terms of a Somos 4 sequence also satisfy

$$
a_{1} a_{2} \tau_{n+m+1} \tau_{n-m}=\left|\begin{array}{ll}
a_{m+1} \tau_{n+2} & a_{m-1} \tau_{n} \\
a_{m+2} \tau_{n+1} & a_{m} \tau_{n-1}
\end{array}\right|
$$

for all $m, n \in \mathbb{Z}$, with $a_{n}$ for $n \in \mathbb{Z}$ given by (1.9) as above. 
Proof. Substituting the formulae (1.9) and (1.12) for $a_{n}$ and $\tau_{n}$ into (1.17), and setting $\hat{z}=z_{0}+\left(n+\frac{1}{2}\right) \kappa$ gives

$$
\begin{aligned}
& \frac{\sigma(\kappa)^{2 m^{2}+2 n^{2}+2 m+2 n+6}}{A^{2} B^{2 n+1}}\left\{a_{1} a_{2} \tau_{n+m+1} \tau_{n-m}-a_{m} a_{m+1} \tau_{n+2} \tau_{n-1}+a_{m-1} a_{m+2} \tau_{n+1} \tau_{n}\right\} \\
& \quad=\sigma(\kappa) \sigma(2 \kappa) \sigma(\hat{z}+(m+1 / 2) \kappa) \sigma(\hat{z}-(m+1 / 2) \kappa) \\
& \quad-\sigma(m \kappa) \sigma((m+1) \kappa) \sigma(\hat{z}+3 \kappa / 2) \sigma(\hat{z}-3 \kappa / 2) \\
& +\sigma((m-1) \kappa) \sigma((m+2) \kappa) \sigma(\hat{z}+\kappa / 2) \sigma(\hat{z}-\kappa / 2)=0
\end{aligned}
$$

by (1.11).

Remarks 1.4. These two corollaries of Theorem 1.1 are the main result of 20, where they are proved by purely algebraic means, i.e., without using explicit analytic expressions such as (1.12). Morgan Ward's formula (1.8) for elliptic divisibility sequences is clearly just a special case of the first corollary. Comparing the statement of Corollary 1.2 when $m=2$ with the expressions for the coefficients of the Somos 4 recurrence in (1.15) gives $\alpha=\left(a_{2}\right)^{2}, \beta=-a_{1} a_{3}$, so it is clearly required that

$$
\wp^{\prime}(\kappa)^{2}=\frac{\sigma(2 \kappa)^{2}}{\sigma(\kappa)^{8}}, \quad \wp^{\prime}(\kappa)^{2}(\wp(2 \kappa)-\wp(\kappa))=-\frac{\sigma(3 \kappa)}{\sigma(\kappa)^{9}} .
$$

To see why these two identities must hold, note that for any $n, a_{n}=\sigma(n \kappa) / \sigma(\kappa)^{n^{2}}$ is an elliptic function of $\kappa$ (see exercise 24 in Chapter 20 of [35]). Thus in the first formula of (1.18), both sides are elliptic functions of $\kappa$ whose Laurent expansions around $\kappa=0$ have the same principal parts; moreover both functions vanish at any one of the half-periods $\kappa=\omega_{j}, j=1,2,3$; hence these two expressions for $\alpha$ are equivalent, and thence the two different formulae for $\beta$ are seen to be equivalent by taking $z=2 \kappa$ in (1.10).

The main results of this paper are found in the next section: Theorem 2.7 presents the explicit solution of a second order nonlinear mapping associated with Somos 5 sequences, while Theorem 2.9 presents the explicit form of the general solution for these sequences. In the third section we briefly illustrate the solution of the initial value problem for such sequences, by applying the theorem to the specific example of the Somos (5) sequence (1.3), which is sequence A006721 in Sloane's catalogue [28].

\section{General solution of Somos 5}

The Somos 4 recurrence has a simple gauge invariance property: if $\tau_{n}$ satisfies (1.4), then for any non-zero constants $\tilde{A}, \tilde{B}$ it is clear that the transformation

$$
\tau_{n} \longrightarrow \tilde{\tau}_{n}=\tilde{A} \tilde{B}^{n} \tau_{n}, \quad n \in \mathbb{Z}
$$

yields another solution of the same fourth order recurrence. Thus it is natural to introduce the gauge-invariant combination

$$
f_{n}=\frac{\tau_{n+1} \tau_{n-1}}{\left(\tau_{n}\right)^{2}}
$$

which satisfies the second order nonlinear mapping defined by

$$
f_{n+1}=\frac{1}{f_{n-1} f_{n}}\left(\alpha+\frac{\beta}{f_{n}}\right)
$$


(written in the more symmetrical form (2.4) below). The following result in [13] was the basis for the proof of Theorem 1.1.

Proposition 2.1. The second order nonlinear mapping defined by

$$
f_{n-1}\left(f_{n}\right)^{2} f_{n+1}=\alpha f_{n}+\beta
$$

has the conserved quantity

$$
J=f_{n-1} f_{n}+\alpha\left(\frac{1}{f_{n-1}}+\frac{1}{f_{n}}\right)+\frac{\beta}{f_{n-1} f_{n}} .
$$

Its general solution has the form

$$
f_{n}=\wp(\kappa)-\wp\left(z_{0}+n \kappa\right)
$$

where $\wp(z)=\wp\left(z ; g_{2}, g_{3}\right)$ is the Weierstrass elliptic function associated with an elliptic curve (1.14) with invariants $g_{2}, g_{3}$. The parameters $z_{0}, \kappa \in \mathbb{C}$ and the two invariants are determined from $\alpha, \beta$ and the initial data $f_{0}, f_{1}$ according to the formulae

$$
\lambda=\frac{1}{3 \alpha}\left(\frac{J^{2}}{4}-\beta\right), \quad g_{2}=12 \lambda^{2}-2 J, \quad g_{3}=4 \lambda^{3}-g_{2} \lambda-\alpha,
$$

together with the elliptic integrals

$$
z_{0}= \pm \int_{\infty}^{\lambda-f_{0}} \frac{\mathrm{d} x}{y}, \quad \kappa= \pm \int_{\infty}^{\lambda} \frac{\mathrm{d} x}{y} .
$$

Remarks 2.2. The above is a slight reformulation of Proposition 2.2 in 13, where the quantity $\lambda=\wp(\kappa)$ was taken as the basic conserved quantity rather than the simpler quantity $J=\wp^{\prime \prime}(\kappa)$; the latter is the same as the "translation invariant" found in Swart's thesis 29. There is an ambiguity in the sign of the integrals (2.8) due to the elliptic involution $y \rightarrow-y$, but the solution (2.6) is clearly invariant under $z_{0} \rightarrow-z_{0}, \kappa \rightarrow-\kappa$ due to the fact that $\wp(z)$ is an even function; the relative sign of $z_{0}$ and $\kappa$ is fixed by the consistency requirement $\wp^{\prime}\left(z_{0}\right) \wp^{\prime}(\kappa)=$ $\left(f_{0}\right)^{2}\left(f_{1}-f_{-1}\right)$. The mapping (2.4), or equivalently (2.3), is of the type studied by Quispel, Roberts and Thompson [23] (see also [1, 14, 15, 30] and references). The solution (2.6) of (2.4) corresponds to the solution (1.12) of the Somos 4 recurrence, modulo the two-parameter abelian group of gauge transformations (2.1).

Proposition 2.3. By means of the substitution (2.2), the Somos 5 recurrence (1.1) corresponds to the third order nonlinear mapping defined by

$$
f_{n-1}\left(f_{n}\right)^{2}\left(f_{n+1}\right)^{2} f_{n+2}=\tilde{\alpha} f_{n} f_{n+1}+\tilde{\beta}
$$

which has two independent conserved quantities $\tilde{I}, \tilde{J}$ given by

$$
\begin{aligned}
& \tilde{I}=f_{n-1} f_{n} f_{n+1}+\tilde{\alpha}\left(\frac{1}{f_{n-1}}+\frac{1}{f_{n}}+\frac{1}{f_{n+1}}\right)+\frac{\tilde{\beta}}{f_{n-1} f_{n} f_{n+1}}, \\
& \tilde{J}=f_{n-1} f_{n}+f_{n} f_{n+1}+\tilde{\alpha}\left(\frac{1}{f_{n-1} f_{n}}+\frac{1}{f_{n} f_{n+1}}\right)+\frac{\tilde{\beta}}{f_{n-1}\left(f_{n}\right)^{2} f_{n+1}} .
\end{aligned}
$$

Any solution of the Somos 4 recurrence (1.4) also satisfies a Somos 5 recurrence (1.1) with parameters

$$
\tilde{\alpha}=-\beta, \quad \tilde{\beta}=\alpha^{2}+\beta J
$$


where $J$ is as in (2.5), and values of the conserved quantities for the associated solution of (2.9) are

$$
\tilde{J}=J, \quad \tilde{I}=2 \alpha .
$$

Proof. Letting $\tilde{I}_{n}$ denote the expression on the right hand side of (2.10), and letting $\tilde{I}_{n+1}$ be the same expression with $n \rightarrow n+1$, we have immediately that $\tilde{I}_{n+1}-\tilde{I}_{n}=0$ whenever (2.9) holds, and similarly $\tilde{J}$ in (2.11) is conserved by the third order nonlinear mapping. Now for any sequence $\tau_{n}, n \in \mathbb{Z}$ that is a solution of a Somos 4 recurrence (1.4), there is a corresponding solution of (2.4) given by (2.2). By considering the expression

$$
\mathcal{E}_{n}:=f_{n-1}\left(f_{n}\right)^{2}\left(f_{n+1}\right)^{2} f_{n+2}-\tilde{\alpha} f_{n} f_{n+1}-\tilde{\beta},
$$

and then using (2.4), first with $n \rightarrow n+1$ to eliminate $f_{n+2}$, and then once more to eliminate $f_{n+1}$, we find

$$
\begin{aligned}
\mathcal{E}_{n} & =\alpha^{2}-\tilde{\beta}+\beta f_{n} f_{n-1}+\beta\left(\frac{\alpha}{f_{n}}-\frac{\tilde{\alpha}}{f_{n} f_{n-1}}\right)-\frac{\alpha \tilde{\alpha}}{f_{n-1}} \\
& =\alpha^{2}+\beta J-\tilde{\beta}-(\beta+\tilde{\alpha})\left(\frac{\alpha}{f_{n-1}}+\frac{\beta}{f_{n-1} f_{n}}\right)
\end{aligned}
$$

(where we have used (2.5) at the last step) and hence $\mathcal{E}_{n}=0$ for all $n$ only when the two conditions (2.12) hold. In that case, $f_{n}$ satisfies (2.9) with these values of $\tilde{\alpha}, \tilde{\beta}$, which is equivalent to $\tau_{n}$ being a solution of (1.1). The values (2.13) follow from similar calculations.

Remark 2.4. Clearly the special case $m=2$ of Corollary 1.2 above means that every Somos 4 is a Somos 5 with $\tilde{\alpha}=a_{3}, \tilde{\beta}=-a_{4} / a_{2}$, so using (1.9) to compare with (1.15) and noting that $J=\wp^{\prime \prime}(\kappa)$ it is clear that the equation $\tilde{\alpha}=a_{3}=-\beta$ is the same as the first equation of (1.18), while the formula for $\tilde{\beta}$ in (2.12) yields the interesting identity

$$
\wp^{\prime}(\kappa)^{4}+\wp^{\prime \prime}(\kappa) \wp^{\prime}(\kappa)^{2}(\wp(2 \kappa)-\wp(\kappa))=-\frac{\sigma(4 \kappa)}{\sigma(2 \kappa) \sigma(\kappa)^{12}} .
$$

The latter should be very tedious to prove by comparing poles and zeros, since on each side the leading term in the Laurent expansion at $\kappa=0$ is $-2 / \kappa^{12}$.

The formula (1.12) is obviously not general enough to capture all of the Somos 5 sequences: not every Somos 5 is a Somos 4! The expression (1.12) only depends on the six parameters $A, B, z_{0}, \kappa, g_{2}, g_{3}$, whereas to specify a generic Somos 5 sequence requires seven parameters, corresponding to the five initial data $\tau_{0}, \tau_{1}$, $\tau_{2}, \tau_{3}, \tau_{4}$ plus the two coefficients $\tilde{\alpha}, \tilde{\beta}$. One way to interpret the extra degree of freedom in Somos 5 sequences is by noting that they have a three-parameter group of gauge transformations given by

$$
\tau_{2 n} \longrightarrow \tilde{\tau}_{2 n}=\tilde{A}_{+} \tilde{B}^{2 n} \tau_{2 n}, \quad \tau_{2 n+1} \longrightarrow \tilde{\tau}_{2 n+1}=\tilde{A}_{-} \tilde{B}^{2 n+1} \tau_{2 n+1}, \quad n \in \mathbb{Z} .
$$

In other words, compared with Somos 4 there is an extra freedom to rescale the even/odd terms differently while leaving the recurrence (1.1) unchanged. The quantity $f_{n}$ defined by (2.2) is not invariant under this larger group of transformations, and thus it is natural to consider something that is, namely

$$
h_{n}=f_{n+1} f_{n}=\frac{\tau_{n+2} \tau_{n-1}}{\tau_{n+1} \tau_{n}} .
$$


Before we consider the recurrence satisfied by $h_{n}$, it is convenient to state the following general result on mappings of the plane associated with biquadratic curves, which have been studied in detail recently by Iatrou and Roberts [14, 15], Bastien and Rogalski [1] and also Tsuda [30].

Proposition 2.5. The quartic curve $\hat{E}$ defined by

$$
\mathcal{B}(X, Y) \equiv e X^{2} Y^{2}+d X Y(X+Y)+c\left(X^{2}+Y^{2}\right)+b(X+Y)+a-K X Y=0
$$

has genus one. The mapping of the plane

$$
\left(u_{n-1}, u_{n}\right) \mapsto\left(u_{n}, u_{n+1}\right)
$$

defined by

$$
u_{n+1} u_{n-1}=\frac{a+b u_{n}+c u_{n}^{2}}{c+d u_{n}+e u_{n}^{2}}
$$

corresponds to the composition of two involutions on $\hat{E}$ and has the conserved quantity

$$
\tilde{K}=e u_{n-1} u_{n}+d\left(u_{n-1}+u_{n}\right)+c\left(\frac{u_{n-1}}{u_{n}}+\frac{u_{n}}{u_{n-1}}\right)+b\left(\frac{1}{u_{n-1}}+\frac{1}{u_{n}}\right)+\frac{a}{u_{n-1} u_{n}} .
$$

The iterates of (2.19) correspond to the sequence of points

$$
\hat{P}_{n}=\left(u_{0}, u_{1}\right)+n \hat{P} \in \hat{E}
$$

for some $\hat{P} \in \hat{E}$. Furthermore, upon uniformizing $\hat{E}$, the coordinates $u_{n}$ can be written as

$$
u_{n}=f\left(z_{0}+n \kappa\right)
$$

in terms of an elliptic function $f$ and certain parameters $z_{0}, \kappa \in \mathbb{C}$.

Proof. To see that the curve $\hat{E}$ is elliptic, it is sufficient to observe that by the transformation $\mathcal{R}=X Y, \mathcal{S}=X+Y$, this symmetric biquadratic is a ramified double cover of the rational curve

$$
e \mathcal{R}^{2}+d \mathcal{R S}+c\left(\mathcal{S}^{2}-2 \mathcal{R}\right)+b \mathcal{S}+a-K \mathcal{R}=0
$$

with branching at four points $(X, X)$ given by the roots of the quartic $\mathcal{B}(X, X)=0$, and so by the Hurwitz formula the genus is one. The symmetric biquadratic (2.17) admits the involutions

$$
F_{1}: \quad(X, Y) \mapsto(Y, X), \quad F_{2}: \quad(X, Y) \mapsto\left(X, Y^{\dagger}\right),
$$

where $y=Y^{\dagger}$ is the unique solution to the quadratic equation $\mathcal{B}(X, y)=0$ apart from $y=Y$. Clearly the composition $G=F_{2} \circ F_{1}$ of these two involutions yields the mapping of the plane (2.18) defined by (2.19), and it is easy to see that the quantity (2.20) is conserved by this mapping, so that $\tilde{K}=K=$ constant and the points $\left(u_{n-1}, u_{n}\right)$ lie on the level set (2.17) for all $n$ (see e.g. 11 for more details of this construction). $G$ is thus a birational map $\hat{E} \rightarrow \hat{E}$ composed of two involutions $F_{j}, j=1,2$. We claim that for all $P \in \hat{E}$ each $F_{j}: P \mapsto 2 C_{j}-P$ for some point $C_{j} \in \hat{E}$, for $j=1,2$ respectively, and hence $G$ is a translation by the point $\hat{P}=2 C_{2}-2 C_{1}$, so $G: P \mapsto P+\hat{P}$ as is required for (2.21) to hold.

To see why the claim is true, note that each involution $F_{j}, j=1,2$ is a birational map $\hat{E} \rightarrow \hat{E}$ with four fixed points. Consider first $F_{1}$, noting that any morphism 
of elliptic curves is a combination of a translation by a point and an isogeny $\phi$ (see [26, p.75). Since $F_{1}$ is birational we require that $\phi \in \operatorname{Aut}(\hat{E})$, and because it is an involution the only possibilities are $\phi=[ \pm 1]$. If $\phi=[1]$, then we must have $F_{1}: P \mapsto P+\Omega$ where $\Omega$ is a non-identity element of the 2 -torsion subgroup (of order four), but then there are no fixed points. Thus it must be the case that $\phi=[-1]$ and so $F_{1}: P \mapsto 2 C_{1}-P$ where the four possible points $C_{1}+\Omega$ (with $\Omega$ being 2-torsion) account for the fixed points, which for $F_{1}$ correspond to the roots of $\mathcal{B}(X, X)=0$. The same argument applies to $F_{2}$.

To obtain the expression (2.22), observe that because $\hat{E}$ has genus one it can be uniformized using elliptic functions; in 35] this result is attributed to Clebsch. Thus we can write $(X, Y)=(g(z), f(z))$ where $g, f$ are rational functions of the Weierstrass functions $\wp(z), \wp^{\prime}(z)$ associated with a curve $E$ in Weierstrass canonical form (1.14) with invariants $g_{2}, g_{3}$, and $z \in \operatorname{Jac}(E)$. Now the map defined by (2.19) corresponds to addition of a point, which is equivalent to a shift $z \mapsto z+\kappa$ on the Jacobian. Then we see that for some $z_{n}$ we have $\left(u_{n-1}, u_{n}\right)=\left(g\left(z_{n}\right), f\left(z_{n}\right)\right)$ and $\left(u_{n}, u_{n+1}\right)=\left(g\left(z_{n+1}\right), f\left(z_{n+1}\right)\right)=\left(g\left(z_{n}+\kappa\right), f\left(z_{n}+\kappa\right)\right)$, whence it follows that $g(z)=f(z-\kappa)$ and $u_{n}=g\left(z_{0}+(n+1) \kappa\right)=f\left(z_{0}+n \kappa\right)$ as required.

Remark 2.6. In essence, the above result for symmetric biquadratic curves was already known to Euler (see Theorem 9 in [32]). A thorough treatment of general integrable maps associated with elliptic curves over an arbitrary field can be found in the recent work [16. For our purposes here, we need to construct the explicit uniformization for particular families of curves of the above type, and the exact form of the corresponding expressions in terms of Weierstrass functions changes when one or another of the parameters in (2.17) degenerates to zero. For example, we see that for the mapping (2.4) associated with Somos 4 sequences, the invariant curve defined by (2.5) in Proposition 2.1 corresponds to the degenerate case of (2.17) obtained by setting $e=1, d=c=0$. In the proof of the analogous result for Somos 5 sequences, we shall see that the relevant mapping corresponds to the case when (2.17) degenerates to a cubic curve by setting $e=c=0$; cf. (2.24) and (2.34) below.

Theorem 2.7. Given a Somos 5 sequence satisfying (1.1), the corresponding quantity $h_{n}$ defined by (2.16) satisfies the second order nonlinear mapping defined by

$$
h_{n-1} h_{n} h_{n+1}=\tilde{\alpha} h_{n}+\tilde{\beta}
$$

which has the conserved quantity

$$
\tilde{J}=h_{n-1}+h_{n}+\tilde{\alpha}\left(\frac{1}{h_{n-1}}+\frac{1}{h_{n}}\right)+\frac{\tilde{\beta}}{h_{n-1} h_{n}} .
$$

The general solution of this map can be written as

$$
\begin{aligned}
h_{n} & =\frac{\sigma\left(z_{0}+(n+2) \kappa\right) \sigma\left(z_{0}+(n-1) \kappa\right)}{\sigma(\kappa)^{4} \sigma\left(z_{0}+n \kappa\right) \sigma\left(z_{0}+(n+1) \kappa\right)} \\
& =-\frac{\wp^{\prime}(\kappa)}{2}\left(\frac{\wp^{\prime}\left(z_{0}+n \kappa\right)-\wp^{\prime}(\kappa)}{\wp\left(z_{0}+n \kappa\right)-\wp(\kappa)}\right)+\frac{\wp^{\prime \prime}(\kappa)}{2},
\end{aligned}
$$

where $\sigma(z)=\sigma\left(z ; g_{2}, g_{3}\right)$ and $\wp(z)=\wp\left(z ; g_{2}, g_{3}\right)$ are Weierstrass functions associated with an elliptic curve

$$
E: \quad y^{2}=4 x^{3}-g_{2} x-g_{3} .
$$


The invariants of the curve are determined from the parameters $\tilde{\alpha}, \tilde{\beta}$ together with the initial data $h_{0}, h_{1}$ for the map (2.23) according to the formulae

$$
\begin{aligned}
\tilde{\mu} & =(\tilde{\beta}+\tilde{\alpha} \tilde{J})^{1 / 4}, & \tilde{\lambda} & =\frac{1}{3 \tilde{\mu}^{2}}\left(\frac{\tilde{J}^{2}}{4}+\tilde{\alpha}\right), \\
g_{2} & =12 \tilde{\lambda}^{2}-2 \tilde{J}, & g_{3} & =4 \tilde{\lambda}^{3}-g_{2} \tilde{\lambda}-\tilde{\mu}^{2} .
\end{aligned}
$$

The parameters $z_{0}, \kappa \in \mathbb{C}$ are then determined by the elliptic integrals

$$
z_{0}= \pm \int_{\infty}^{x_{0}} \frac{\mathrm{d} x}{y}, \quad \kappa= \pm \int_{\infty}^{\tilde{\lambda}} \frac{\mathrm{d} x}{y}
$$

on the curve $E$, where

$$
x_{0}=\tilde{\lambda}+\frac{\tilde{\mu}^{2}}{h_{-1}+h_{0}-\tilde{J}}, \quad h_{-1}=\frac{\tilde{\alpha} h_{0}+\tilde{\beta}}{h_{0} h_{1}}
$$

and the relative sign of $\kappa$ and $z_{0}$ is fixed by requiring the consistency of

$$
\tilde{\mu}=\wp^{\prime}(\kappa), \quad \wp^{\prime}(\kappa) \wp^{\prime}\left(z_{0}\right)=\left(x_{0}-\tilde{\lambda}\right)\left(h_{-1}-h_{0}\right) .
$$

Proof. Letting $\tilde{J}_{n}$ denote the right hand side of (2.24), it is clear that

$$
\tilde{J}_{n+1}-\tilde{J}_{n}=\frac{\left(h_{n+1}-h_{n-1}\right)}{h_{n-1} h_{n} h_{n-1}}\left(h_{n+1} h_{n} h_{n-1}-\tilde{\alpha} h_{n}-\tilde{\beta}\right),
$$

so assuming that $h_{n+1} \neq h_{n-1}$ for all $n$, then $\tilde{J}$ is conserved if and only if $h_{n}$ satisfies (2.23). Alternatively note that rewriting (2.11) in terms of $h_{n}$ gives precisely (2.24), and rearranging the latter expression for $\tilde{J}$ yields

$$
\left(h_{n-1}+h_{n}-\tilde{J}\right)\left(h_{n-1} h_{n}+\tilde{\alpha}\right)+\tilde{\beta}+\tilde{\alpha} \tilde{J}=0,
$$

which implies that for any sequence $h_{n}, n \in \mathbb{Z}$ satisfying (2.23), $(X, Y)=\left(h_{n-1}, h_{n}\right)$ is a point on the curve

$$
(X+Y-\tilde{J})(X Y+\tilde{\alpha})+\tilde{\beta}+\tilde{\alpha} \tilde{J}=0
$$

for all $n$.

We claim that this elliptic curve is birationally equivalent to (2.26) via the transformation

$$
X=\frac{\tilde{\mu}}{2}\left(\frac{y+\tilde{\mu}}{x-\tilde{\lambda}}\right)+\frac{\tilde{J}}{2}, \quad Y=-\frac{\tilde{\mu}}{2}\left(\frac{y-\tilde{\mu}}{x-\tilde{\lambda}}\right)+\frac{\tilde{J}}{2},
$$

where the parameters $\tilde{\lambda}, \tilde{\mu}$ and invariants $g_{2}, g_{3}$ are found from the coefficients of the curve (2.34) using the formulae (2.27) and (2.28). To verify this, it is instructive to use the analytic formulae (2.25) which provide a uniformization of (2.34). First note that the two formulae for $h_{n}$ in (2.25) are easily seen to be equivalent by noting that both expressions are elliptic functions of $z_{0}$ with simple zeros at $z_{0}=-(n+2) \kappa$, $-(n-1) \kappa$ and simple poles at $z_{0}=-n \kappa,-(n+1) \kappa$, with the same residues at these poles. Similarly, shifting $n \rightarrow n-1$ in the first (sigma function) expression in (2.25) gives

$$
\begin{aligned}
h_{n-1} & =\frac{\sigma\left(z_{0}+(n+1) \kappa\right) \sigma\left(z_{0}+(n-2) \kappa\right)}{\sigma(\kappa)^{4} \sigma\left(z_{0}+(n-1) \kappa\right) \sigma\left(z_{0}+n \kappa\right)} \\
& =\frac{\wp^{\prime}(\kappa)}{2}\left(\frac{\wp^{\prime}\left(z_{0}+n \kappa\right)+\wp^{\prime}(\kappa)}{\wp\left(z_{0}+n \kappa\right)-\wp(\kappa)}\right)+\frac{\wp^{\prime \prime}(\kappa)}{2},
\end{aligned}
$$


where once again these two formulae for $h_{n-1}$ are seen to be equivalent by looking at their poles and zeros. Then we can make the identifications $(2.37)$

$\tilde{\lambda}=\wp(\kappa), \quad \tilde{\mu}=\wp^{\prime}(\kappa)=(\tilde{\beta}+\tilde{\alpha} \tilde{J})^{1 / 4}, \quad \tilde{J}=\wp^{\prime \prime}(\kappa), \quad \tilde{\alpha}=-\wp^{\prime}(\kappa)^{2}(\wp(2 \kappa)-\wp(\kappa))$,

which are consistent with (2.27) and (2.28); i.e., $(\tilde{\lambda}, \tilde{\mu})=\left(\wp(\kappa), \wp^{\prime}(\kappa)\right)$ is a point on the curve (2.26).

Taking the sum of the right hand ( $\wp$ function) expressions in (2.25) and (2.36) gives

$$
h_{n-1}+h_{n}=\wp^{\prime \prime}(\kappa)+\frac{\wp^{\prime}(\kappa)^{2}}{\wp\left(z_{0}+n \kappa\right)-\wp(\kappa)},
$$

which by (2.37) is equivalent to $X+Y=\tilde{J}+\tilde{\mu}^{2} /(x-\tilde{\lambda})$ with the identification $(x, y)=\left(\wp\left(z_{0}+n \kappa\right), \wp^{\prime}\left(z_{0}+n \kappa\right)\right)$. On the other hand, taking the product of the left hand (sigma function) expressions in (2.25) and (2.36) gives

$$
h_{n-1} h_{n}=\frac{\sigma\left(z_{0}+(n+2) \kappa\right) \sigma\left(z_{0}+(n-2) \kappa\right)}{\sigma(\kappa)^{8} \sigma\left(z_{0}+n \kappa\right)^{2}}=\wp^{\prime}(\kappa)^{2}\left(\wp(2 \kappa)-\wp\left(z_{0}+n \kappa\right)\right),
$$

where we have used (1.10) and the first identity in (1.18). With the same identifications as before, this product identity implies that we have $X Y+\tilde{\alpha}=-\tilde{\mu}^{2}(x-\tilde{\lambda})$, and hence $(X+Y-\tilde{J})(X Y+\tilde{\alpha})=-\tilde{\mu}^{4}=-(\tilde{\beta}+\tilde{\alpha} \tilde{J})$, which means that $(X, Y)=$ $\left(h_{n-1}, h_{n}\right)$ lies on the curve (2.34) for any $n$, as claimed. In turn this means that the sequence $\left\{h_{n} \mid n \in \mathbb{Z}\right\}$ defined by (2.25) has $\tilde{J}=\wp^{\prime \prime}(\kappa)$ as a conserved quantity, and hence by (2.32) it must satisfy the second order map (2.23).

To see that (2.25) represents the unique solution of the initial value problem for the map (2.23), observe that it depends on the four parameters $g_{2}, g_{3}, z_{0}, \kappa$. Up to the involution $z_{0} \rightarrow-z_{0}, \kappa \rightarrow-\kappa$ (which does not change the solution), all of the latter are uniquely determined from the initial data $h_{0}, h_{1}$ and parameters $\tilde{\alpha}$, $\tilde{\beta}$ by first constructing the first integral $\tilde{J}=\tilde{J}_{0}$ (i.e., setting $n=0$ in (2.24) $)$ and then using the formulae (2.27) and (2.28) to find the invariants. Finally the base point $z_{0}$ and the shift $\kappa$ on the Jacobian $\operatorname{Jac}(E)$ of the curve (2.26) are obtained by inverting $x_{0}=\wp\left(z_{0}\right), \tilde{\lambda}=\wp(\kappa)$ to yield the elliptic integrals (2.29), where $x_{0}$ in (2.30) is found by rearranging (2.38) for $n=0$. The consistency requirements (2.31) follow from fixing the branch of the fourth root taken for $\tilde{\mu}$ in (2.27) and ensuring that the value of $\wp^{\prime}\left(z_{0}\right)$ agrees with this in the formulae for $h_{0}$ and $h_{-1}$ (that is, the $\wp$ function expressions in (2.25) and (2.36) when $n=0$ ).

Proposition 2.8. For any solution $\tau_{n}, n \in \mathbb{Z}$ of a Somos 5 recurrence (1.1), the subsequences $\tau_{n}^{*}=\tau_{2 n}$ and $\tau_{n}^{*}=\tau_{2 n+1}$, of respectively even and odd index terms, both satisfy the Somos 4 recurrence

$$
\tau_{n+2}^{*} \tau_{n-2}^{*}=\alpha^{*} \tau_{n+1}^{*} \tau_{n-1}^{*}+\beta^{*}\left(\tau_{n}^{*}\right)^{2},
$$

where

$$
\alpha^{*}=\tilde{\beta}^{2}, \quad \beta^{*}=\tilde{\alpha}\left(2 \tilde{\beta}^{2}+\tilde{\alpha} \tilde{\beta} \tilde{J}+\tilde{\alpha}^{3}\right),
$$

with $\tilde{J}$ being the associated integral (2.24) of the map (2.23).

Proof. Looking at the formula (2.39) we see that

$$
h_{n} h_{n-1}=f_{n+1}\left(f_{n}\right)^{2} f_{n-1}=\frac{\tau_{n+2} \tau_{n-2}}{\left(\tau_{n}\right)^{2}}=\wp^{\prime}(\kappa)^{2}\left(\wp(2 \kappa)-\wp\left(z_{0}+n \kappa\right)\right) \text {, }
$$


which is almost identical to the formula (2.6) for the solution of the map (2.4), except for the scale factor $\tilde{\mu}^{2}=\wp^{\prime}(\kappa)^{2}=\sqrt{\tilde{\beta}+\tilde{\alpha} \tilde{J}}$. Using the scaling property

$$
\wp\left(z ; g_{2}, g_{3}\right)=\tilde{\mu}^{-2} \wp\left(z / \tilde{\mu} ; \tilde{\mu}^{4} g_{2}, \tilde{\mu}^{6} g_{3}\right), \quad \sigma\left(z ; g_{2}, g_{3}\right)=\tilde{\mu} \sigma\left(z / \tilde{\mu} ; \tilde{\mu}^{4} g_{2}, \tilde{\mu}^{6} g_{3}\right),
$$

of the Weierstrass functions, we can rewrite everything in terms of rescaled sigma and $\wp$ functions with invariants $g_{2}^{*}=\tilde{\mu}^{4} g_{2}, g_{3}^{*}=\tilde{\mu}^{6} g_{3}$, setting $v=\kappa / \tilde{\mu}$ and $u_{0}=$ $z_{0} / \tilde{\mu}$ to obtain the canonical form

$$
\frac{\tau_{n+2} \tau_{n-2}}{\left(\tau_{n}\right)^{2}}=\wp(2 v)-\wp\left(u_{0}+n v\right)=\frac{\sigma\left(u_{0}+(n+2) v\right) \sigma\left(u_{0}+(n-2) v\right)}{\sigma\left(u_{0}+n v\right)^{2} \sigma(2 v)^{2}} .
$$

It follows immediately from Proposition 2.1 that both $F_{n}^{*}=\tau_{2 n+2} \tau_{2 n-2} /\left(\tau_{2 n}\right)^{2}$ and $F_{n}^{*}=\tau_{2 n+3} \tau_{2 n-1} /\left(\tau_{2 n+1}\right)^{2}$ are solutions of the nonlinear map given by

$$
F_{n+1}\left(F_{n}\right)^{2} F_{n-1}=\alpha^{*} F_{n}+\beta^{*}
$$

with

$$
\alpha^{*}=\wp^{\prime}(2 v)^{2}, \quad \beta^{*}=\wp^{\prime}(2 v)^{2}(\wp(4 v)-\wp(2 v)) .
$$

The required result follows immediately, upon noting that the duplication formula for the Weierstrass $\wp$ function can be used in (2.43) to rewrite $\wp(4 v)$ in terms of $\wp(2 v)$ and its derivatives, and similarly for $\wp(2 v)$ in terms of $\wp(v)$. After further tedious computations, scaling back to the original Weierstrass functions $\tilde{\alpha}=\wp(\kappa)$, $\tilde{\mu}=\wp^{\prime}(\kappa), \tilde{J}=\wp^{\prime \prime}(\kappa)$ with invariants $g_{2}, g_{3}$, we arrive at the expressions (2.41).

Theorem 2.9. The general solution of the Somos 5 recurrence takes the alternating form

$$
\tau_{2 n}=A_{+} B_{+}^{n} \frac{\sigma\left(u_{0}+2 n v\right)}{\sigma(2 v)^{n^{2}}}, \quad \tau_{2 n+1}=A_{-} B_{-}^{n} \frac{\sigma\left(u_{0}+(2 n+1) v\right)}{\sigma(2 v)^{n^{2}}},
$$

where here $\sigma(u)=\sigma\left(u ; g_{2}^{*}, g_{3}^{*}\right)$ denotes the Weierstrass sigma function associated with the elliptic curve

$$
E^{*}: \quad y^{2}=4 x^{3}-g_{2}^{*} x-g_{3}^{*},
$$

where $u_{0}, v$ and the invariants are given in terms of the parameters (2.27), (2.28) in Theorem 2.7 by

$$
g_{2}^{*}=\tilde{\mu}^{4} g_{2}, \quad g_{3}^{*}=\tilde{\mu}^{6} g_{3}, \quad u_{0}=z_{0} / \tilde{\mu}, \quad v=\kappa / \tilde{\mu},
$$

and the prefactors are

$$
\begin{array}{cc}
A_{+}=\frac{\tau_{0}}{\sigma\left(u_{0}\right)}, & A_{-}=\frac{\tau_{1}}{\sigma\left(u_{0}+v\right)}, \\
B_{+}=\frac{\sigma(2 v) \sigma\left(u_{0}\right) \tau_{2}}{\sigma\left(u_{0}+2 v\right) \tau_{0}}, & B_{-}=\frac{\sigma(2 v) \sigma\left(u_{0}+v\right) \tau_{3}}{\sigma\left(u_{0}+3 v\right) \tau_{1}} .
\end{array}
$$

The solution (2.44) depends on seven independent parameters $g_{2}^{*}, g_{3}^{*}, u_{0}, v, A_{+}, A_{-}$ and $B_{+}$, with $B_{ \pm}$defined in (2.47) being related by

$$
\frac{B_{+}}{B_{-}}=-\sigma(2 v)
$$


Proof. Starting from the result of Proposition 2.8, the formula (2.42) for the ratios of the $\tau_{n}$ can be used to solve for the even and odd index subsequences separately, since these are both Somos 4 sequences. In that case, Theorem 1.1 applies separately to the two subsequences $\tau_{2 n}$ and $\tau_{2 n+1}$, which yields (2.44); the proof for each subsequence is the same as in 13 . However, the solution can depend on at most 7 parameters, as is confirmed by the relation (2.48) between $B_{ \pm}$. To prove the latter relationship, it suffices to rewrite the ratios of sigmas and $\tau_{n}$ in terms of $h_{1}$, and see that most terms cancel, taking care to scale appropriately with

$$
\tilde{\mu}=\wp^{\prime}\left(\kappa ; g_{2}, g_{3}\right), \quad \tilde{\mu}^{4}=\wp^{\prime}\left(v ; g_{2}^{*}, g_{3}^{*}\right) .
$$

Remark 2.10. The rescaled curve (2.45) is more convenient when working in fields other than $\mathbb{C}$, since then one does not need to define the curve over the field extension by the fourth $\operatorname{root} \tilde{\mu}=(\tilde{\beta}+\tilde{\alpha} \tilde{J})^{1 / 4}$.

Corollary 2.11. The solution of the third order nonlinear map (2.9) can be written in the alternating form

$$
f_{2 n}=f_{0}\left(\frac{\wp(v)-\wp\left(u_{0}+2 n v\right)}{\wp(v)-\wp\left(u_{0}\right)}\right), \quad f_{2 n+1}=f_{1}\left(\frac{\wp(v)-\wp\left(u_{0}+(2 n+1) v\right)}{\wp(v)-\wp\left(u_{0}+v\right)}\right),
$$

where $\wp(u)=\wp\left(u ; g_{2}^{*}, g_{3}^{*}\right)$ is the Weierstrass $\wp$ function associated with the curve (2.45), with the parameters $u_{0}, v$ as given in Theorem 2.9.

Proof. This follows by substituting (2.44) into (2.2) and rewriting suitable combinations of the prefactors $A_{ \pm}, B_{ \pm}$in terms of $f_{0}$ and $f_{1}$.

Corollary 2.12. The terms $\tau_{n}, n \in \mathbb{Z}$ of a Somos 5 sequence satisfy the Hankel determinant formula

$$
a_{1} a_{2} \tau_{n+m+1} \tau_{n-m}=\left|\begin{array}{ll}
a_{m+1} \tau_{n+2} & a_{m-1} \tau_{n} \\
a_{m+2} \tau_{n+1} & a_{m} \tau_{n-1}
\end{array}\right|
$$

for all $m, n \in \mathbb{Z}$, where the quantities $a_{m}$, which form an associated elliptic divisibility sequence, are given by

$$
a_{m}=\frac{\sigma(m \kappa)}{\sigma(\kappa)^{m^{2}}}, \quad m \in \mathbb{Z}
$$

in terms of the sigma function $\sigma(z)=\sigma\left(z ; g_{2}, g_{3}\right)$ for the curve (2.26) in Theorem 2.7.

Proof. Using the scaling by $\tilde{\mu}$ as in (2.49), the formulae (2.44) can be rewritten as

$$
\tau_{2 n}=A_{+} B_{+}^{n} \frac{\tilde{\mu}^{n^{2}-1} \sigma\left(z_{0}+2 n \kappa\right)}{\sigma(2 \kappa)^{n^{2}}}, \tau_{2 n+1}=A_{-} B_{-}^{n} \frac{\tilde{\mu}^{n^{2}-1} \sigma\left(z_{0}+(2 n+1) \kappa\right)}{\sigma(2 \kappa)^{n^{2}}},
$$

in terms of the sigma function for the original curve (2.26) as in Theorem 2.7. In terms of this sigma function it is easy to show, upon rescaling (2.48) by $\tilde{\mu}=$ $\wp^{\prime}\left(\kappa ; g_{2}, g_{3}\right)=-\sigma\left(2 \kappa ; g_{2}, g_{3}\right) / \sigma\left(\kappa ; g_{2}, g_{3}\right)^{4}$ (compare with the first formula of (1.18) $)$, that

$$
B_{+} / B_{-}=\sigma\left(\kappa ; g_{2}, g_{3}\right)^{4} .
$$

For verification of (2.51), the symmetry $m \rightarrow-m-1$ means that it is sufficient to check only the cases when $m, n$ are either both odd or both even, to take the 
different expressions (2.52) for $\tau_{n}$ with odd/even $n$ into account. Thereafter the proof is the same as for Corollary 1.3.

Corollary 2.13. The terms of a Somos 5 sequence, satisfying the recurrence (1.1), have the leading order asymptotic behaviour

$$
\log \left|\tau_{n}\right| \sim\left(\operatorname{Re}\left\{\frac{\eta_{1} v^{2}}{2 \omega_{1}}\right\}-\frac{1}{4} \log |\sigma(2 v)|\right) n^{2}, \quad n \rightarrow \infty
$$

for $v \in \omega_{1} \mathbb{R}$, where $\omega_{1}$ is a half-period and $\eta_{1}=\zeta\left(\omega_{1}\right)$, with the invariants $g_{2}^{*}$, $g_{3}^{*}$ being as in Theorem 2.9.

Proof. The sigma function is given by the product formula

$$
\sigma(z)=\frac{2 \omega_{1}}{\pi} \exp \left(\frac{\eta_{1} z^{2}}{2 \omega_{1}}\right) \sin \left(\frac{\pi z}{2 \omega_{1}}\right) \prod_{n=1}^{\infty}\left\{\frac{1-2 q^{2 n} \cos \left(\pi z / \omega_{1}\right)+q^{4 n}}{\left(1-q^{2 n}\right)^{2}}\right\}
$$

with $q=\exp \left(\pi i \omega_{2} / \omega_{1}\right)$ (see $\S 20.421$ and also $\S 21.43$ in 35 for the corresponding theta function expression). The result then follows upon taking the logarithm of (2.44). The corrections to (2.53) at $O(n)$ can be calculated similarly, although these have a different form for even/odd $n$.

\section{Example: Somos (5)}

To illustrate the above results, we consider the example of the Somos (5) sequence (1.3). The initial data and parameters as in (1.2) give $h_{0}=2, h_{1}=1$ for the second order map (2.23), whence $h_{-1}=3 / 2$ with $\tilde{\alpha}=1=\tilde{\beta}$, so applying the formulae in Theorem 2.5 we have

$$
\tilde{J}=5, \quad \tilde{\mu}=6^{1 / 4}, \quad \tilde{\lambda}=\frac{29}{12 \sqrt{6}}, \quad x_{0}=-\frac{19}{12 \sqrt{6}},
$$

whence $g_{2}=121 / 72, g_{3}=-845 /(1296 \sqrt{6})$. Rescaling by appropriate factors of $\tilde{\mu}$, as in Theorem 2.9, we arrive at the curve

$$
E^{*}: \quad y^{2}=4 x^{3}-\frac{121}{12} x+\frac{845}{216}, \quad g_{2}^{*}=\frac{121}{12}, \quad g_{3}^{*}=-\frac{845}{216},
$$

which has the $j$-invariant

$$
j=\frac{1728\left(g_{2}^{*}\right)^{3}}{\left(g_{2}^{*}\right)^{3}-27\left(g_{3}^{*}\right)^{2}}=\frac{1771561}{612} .
$$

Hence the Somos (5) sequence (1.3) corresponds to the sequence of points

$$
\left(x_{0}^{*}, y_{0}^{*}\right)+n\left(\lambda^{*}, \mu^{*}\right)=(-19 / 12,2)+n(29 / 12,6)=(5 / 12,0)+(n-2)(29 / 12,6)
$$

on the rescaled curve (3.1).

For comparison with results of other authors, we note that van der Poorten [19] has obtained the Somos (5) sequence from the minimal model $V^{2}+U V+6 V=U^{3}+$ $7 U^{2}+12 U$, while Zagier [36] found the related curve $y^{2}+x y=x(x-1)(x+2)$; both of these curves have the same $j$-invariant as (3.1), and hence they are birationally equivalent to each other. Zagier's discussion makes use of the inspired substitution (2.16) for the Somos (5) sequence (1.3), although the presentation of the associated curve in the form (2.34) contains a typographical error in 36]. Other approaches to the sequence (1.3) can be found on postings to Propp's "robbins" forum [22]; see, in particular, the references to unpublished work of Elkies in [5], where the sequence (1.3) is related to the problem of Heron triangles with two rational medians. 
With the curve (3.1) and the sequence of points (3.2) we can use Theorem 2.9 to calculate the general term of the Somos 5 recurrence in the form (2.44) with

$$
u_{0}=0.163392411+\omega_{2}, \quad v=-0.672679183,
$$

where the real and imaginary half-periods are

$$
\omega_{1}=1.181965956, \quad \omega_{2}=0.973928783 i
$$

respectively (doing all the elliptic integrals to 9 decimal places with MAPLE). The result of Corollary 2.13 then implies that the terms of the sequence (1.3) have the leading order asymptotics

$$
\log \tau_{n} \sim 0.071626946 n^{2}, \quad n \rightarrow \infty .
$$

\section{Conclusions}

The theorems presented in [13] and above provide a simple algorithmic approach to determining elliptic curves associated with Somos 4 and Somos 5 sequences, and finding the complete solution of the associated initial value problems. Our approach is based on making the connection with explicitly solvable maps with conserved quantities, i.e., (2.4) and (2.23). A symplectic map with sufficiently many conserved quantities is called integrable 3 , 31. As they stand the maps of the plane presented here are symplectic in log-canonical coordinates; by making a change of variables, another symplectic structure was given in [13.

The results presented above naturally lead to the question of how to solve Somos $(2 N+2)$ recurrences with $N+2$ terms, of the form

$$
\tau_{n+N+1} \tau_{n-N-1}=\sum_{j=0}^{N} \alpha_{j} \tau_{n+j} \tau_{n-j},
$$

with constant coefficients $\alpha_{j}$, and similarly for Somos $(2 N+3)$. By using the continued fraction expansion of the square root of a sextic, van der Poorten [21] has generated a certain class of Somos 6 sequences associated with genus 2 curves. With a different approach [2] based on the addition formulae for Kleinian sigma functions (see [6] and references), we have constructed Somos 8 sequences derived from genus 2 curves, taking a different (quintic) affine model compared with van der Poorten. The recurrences in [2] generalise the genus 2 case of Cantor's hyperelliptic division polynomials [7] (for analytic formulae, see [17]), and they are related to a family of integrable symplectic maps (discrete Hénon-Heiles systems). We should also remark that Buchstaber and Krichever have derived bilinear addition formulae for Riemann theta functions 4, which have exactly $N+2$ terms in genus $N$. Whether this result can lead to an effective solution to the initial value problem for these higher order recurrences is the subject of further investigation. It would also be interesting to see how some of our results on Somos 4 and 5 sequences could be modified in the $p$-adic setting 27 .

Acknowledgments. I am grateful to Graham Everest for introducing me to the arithmetic of quadratic recurrence sequences, to Christine Swart for sending me her thesis [29], and to Marc Rogalski for sending me his work [1]. Thanks also to Tom Ward, David Cantor and Alf van der Poorten for interesting correspondence on related matters, and to the University of Kent for supporting the project Algebraic curves and functional equations in mathematical physics with a Colyer-Fergusson Award. Finally, I would like to thank the referee for suggesting Proposition 2.5. 


\section{REFERENCES}

1. G. Bastien and M. Rogalski, On some algebraic difference equations $u_{n+2} u_{n}=\psi\left(u_{n+1}\right)$ in $\mathbb{R}_{*}^{+}$, related to families of conics or cubics: Generalization of the Lyness' sequences, J. Math. Anal. Appl. 300 (2004) 303-333. MR2098211 (2005j:39005)

2. H.W. Braden, V.Z. Enolskii and A.N.W. Hone, Bilinear recurrences and addition formulae for hyperelliptic sigma functions, Journal of Nonlinear Mathematical Physics 12 (2005), 46-62. MR 2217095 (2007a:14041)

3. M. Bruschi, O. Ragnisco, P.M. Santini and G.-Z. Tu, Integrable Symplectic Maps, Physica D 49 (1991), 273-294. MR1115864 (92g:58031)

4. V.M. Buchstaber and I.M. Krichever, Vector Addition Theorems and Baker-Akhiezer Functions, Teoret. Mat. Fiz. 94 (1993), 200-212. MR1221731 (94h:14047)

5. R.H. Buchholz and R.L. Rathbun, An Infinite Set of Heron Triangles with Two Rational Medians, Amer. Math. Monthly 104 (1997) 107-115. MR1437411(98a:51015)

6. V.M. Buchstaber, V.Z. Enolskii and D.V. Leykin, Hyperelliptic Kleinian functions and applications, in 'Solitons, Geometry and Topology: On the Crossroad,' (eds. V.M. Buchstaber and S.P. Novikov), AMS Translations Series 2, Vol. 179, Amer. Math. Soc., Providence, RI, 1997, pp. 1-33. MR1437155 (98b:14029)

7. D. Cantor, On the analogue of the division polynomials for hyperelliptic curves, J. reine angew. Math. 447 (1994), 91-145. MR1263171 (94m:11071)

8. M. Einsiedler, G. Everest and T. Ward, Primes in elliptic divisibility sequences, LMS Journal of Computation and Mathematics 4 (2001), 1-13. MR1815962 (2002e:11181)

9. G. Everest, V. Miller and N. Stephens, Primes generated by elliptic curves, Proc. Amer. Math. Soc. 132 (2003), 955-963. MR2045409(2005a:11076)

10. G. Everest, A. van der Poorten, I. Shparlinski and T. Ward, Recurrence Sequences, AMS Mathematical Surveys and Monographs, vol. 104, Amer. Math. Soc., Providence, RI, 2003. MR.1990179 (2004c:11015)

11. S. Fomin and A. Zelevinsky, The Laurent Phenomenon, Adv. Appl. Math. 28 (2002), 119-144. MR 1888840 (2002m:05013)

12. D. Gale, The strange and surprising saga of the Somos sequences, Mathematical Intelligencer 13 (1) (1991), 40-42.

13. A.N.W. Hone, Elliptic curves and quadratic recurrence sequences, Bull. London Math. Soc. 37 (2) (2005) 161-171; Corrigendum, Bull. London Math. Soc. 38 (2006) 741-742. MR 2119015 (2005h:11111) MR 2268357

14. A. Iatrou and J.A.G. Roberts, Integrable mappings of the plane preserving biquadratic invariant curves, J. Phys. A: Math. Gen. 34 (2001) 6617-6636. MR.1873990(2003b:37086)

15. A. Iatrou and J.A.G. Roberts, Integrable mappings of the plane preserving biquadratic invariant curves II, Nonlinearity 15 (2002) 459-489. MR1888861 (2003b:37087)

16. D. Jogia, J.A.G. Roberts and F. Vivaldi, An algebraic geometric approach to integrable maps of the plane, J. Phys. A 39 (2006) 1133-1149. MR2200430 (2006k:14055)

17. S. Matsutani, Recursion relation of hyperelliptic PSI-functions of genus two, Int. Transforms Spec. Func. 14 (2003) 517-527. MR2017658 (2004m:14097)

18. R. Miranda, Algebraic Curves and Riemann Surfaces, American Mathematical Society (1995). MR.1326604 (96f:14029)

19. A.J. van der Poorten, Elliptic curves and continued fractions, J. Integer Sequences 8 (2005) Article 05.2.5. MR.2152285 (2006h:11083)

20. A.J. van der Poorten and C.S. Swart, Recurrence Relations for Elliptic Sequences: Every Somos 4 is a Somos k, Bull. London Math. Soc. 38 (2006) 546-554. MR2250745

21. A.J. van der Poorten, Curves of genus 2, continued fractions and Somos Sequences, J. Integer Seq. 8 (2005), Article 05.3.4.

22. J. Propp, The "bilinear" forum, and The Somos Sequence Site, http://www.math.wisc. edu/ propp/

23. G.R.W. Quispel, J.A.G. Roberts and C.J. Thompson, Integrable mappings and soliton equations II, Physica D 34 (1989), 183-192. MR982386 (90e:58066)

24. R. Robinson, Periodicity of Somos sequences, Proc. Amer. Math. Soc. 116 (1992) 613-619. MR 1140672 (93a:11012)

25. R. Shipsey, Elliptic divisibility sequences, Ph.D. thesis, University of London (2000).

26. J.H. Silverman, The Arithmetic of Elliptic Curves, Springer (1986). MR817210 (87g:11070) 
27. J.H. Silverman, p-adic properties of division polynomials and elliptic divisibility sequences, Math. Annal. 332 (2005) 443-471; Addendum 473-474. MR2178070 (2006f:11063)

28. N.J.A. Sloane, On-Line Encyclopedia of Integer Sequences, http://www.research.att.com $/ \sim n j a s / s e q u e n c e s$, sequence A006721.

29. C.S. Swart, Elliptic curves and related sequences, Ph.D. thesis, University of London (2003).

30. T. Tsuda, Integrable mappings via rational elliptic surfaces, J. Phys. A: Math. Gen. 37 (2004) 2721-2730. MR2047557 (2004m:14078)

31. A.P. Veselov, Integrable maps, Russian Math. Surveys 46 (1991), 1-51. MR.1160332 (93e:58096)

32. A.P. Veselov, What Is an Integrable Mapping?, in What Is Integrability?, V.E. Zakharov (ed.), Springer-Verlag (1991) 251-272. MR.1098340 (92c:58119)

33. M. Ward, Memoir on Elliptic Divisibility Sequences, Amer. J. Math. 70 (1948), 31-74. MR.0023275 (9:332j)

34. M. Ward, The Law of Repetition of Primes in an Elliptic Divisibility Sequence, Duke Math. J. 15 (1948), 941-946. MR0027286 (10:283e)

35. E.T. Whittaker and G.N. Watson, A Course of Modern Analysis, 4th edition, Cambridge, 1965.

36. D. Zagier, 'Problems posed at the St. Andrews Colloquium, 1996,' Solutions, 5th day; available at http://www-groups.dcs.st-and.ac.uk/ john/Zagier/Problems.html

Institute of Mathematics, Statistics and Actuarial Science, University of Kent, Canterbury CT2 7NF, United Kingdom

E-mail address: anwh@kent.ac.uk 